\title{
Guillain-Barré Syndrome Complicating First Trimester Pregnancy: A Case Report and Review of Literature
}

\author{
Aniska W Fortuin ${ }^{1 \#}\left(\mathbb{D}\right.$, Anne-Lotte F van der Kooi ${ }^{2 \#}\left(\mathbb{D}\right.$, Laus JMM Mulder ${ }^{1}$ and Irene AM \\ van der Avoort ${ }^{2 *}$ (D)
}

${ }^{1}$ Department of Neurology, Ikazia Hospital, The Netherlands

${ }^{2}$ Department of Obstetrics and Gynaecology, Ikazia Hospital, The Netherlands

*Corresponding author: IAM van der Avoort, Department of Obstetrics and Gynaecology, Ikazia Hospital, Montessoriweg 1, 3083, AN, Rotterdam, The Netherlands

\begin{abstract}
Background: Guillain-Barre syndrome (GBS) is a rare immune mediated polyradiculoneuropathy, causing muscle weakness, hyporeflexia and dysesthesia. There is no specific guideline for management of GBS during pregnancy in general and first term in particular.
\end{abstract}

Objectives: We report a 26-year-old nullipara who developed GBS in early pregnancy and present an overview of recommended treatment, maternal and fetal outcomes and pathogens associated with GBS in women diagnosed in first trimester pregnancy.

Methods: We performed a systematic literature search using Embase and Web of Science databases. Eligible publications included all English reports of the last twenty years describing GBS diagnosed in the first trimester of pregnancy.

Results: The literature search showed 89 unique articles of which four fulfilled our inclusion criteria. Intravenous immunoglobulins (IVIG) were the treatment of choice administered to the pregnant women diagnosed with GBS. All women recovered completely. Two articles report Cytomegalovirus (CMV) infection, leading to congenital infection in one case and in utero fetal death in another. One woman delivered a healthy baby after receiving mechanical ventilation for 18 weeks and one woman had a miscarriage. We present a case diagnosed with GBS without known causative agent, who recovered after IVIG treatment and delivered a healthy neonate at term.

Conclusion: The treatment of GBS in pregnant patients is similar to the treatment of other patients and consists of IVIG and/or plasmapheresis and supportive care. The severity of polyradiculopathy seems to be unrelated to fetal outcomes, in contrast to the causative infection. CMV infection should be evaluated as causative agent.

\section{Keywords}

Guillain Barré syndrome, Pregnancy, Cytomegalovirus, Polyradiculoneuropathy

\section{Introduction}

Guillain-Barré syndrome (GBS) is a rare immune mediated neuropathy, causing symmetrical muscle weakness, absence of muscle tendon reflexes and dysesthesia. Symptoms range from minimal distal muscle weakness to severe respiratory insufficiency with the need of mechanical ventilation. When autonomic nerves are affected, autonomic dysregulation (i.e. cardiac arrhythmias or blood pressure lability) can occur. In the acute stadium, GBS has a mortality of less than $5 \%$, but about $20 \%$ of patients continue to have significant disabilities [1]. The pathophysiology is based on molecular mimicry, a mechanism by which infectious agents may trigger an immune response against auto antigens [2]. Campylobacter Jejuni is a well-known trigger antigen, but GBS also has been reported following infection with Mycoplasma pneumoniae, Haemophilus influenzae, cytomegalovirus, Epstein-Barr virus and, as recently discovered, Zika virus [3,4]. Patients often mention an antecedent illness in the 1-6 weeks preceding the development of neurological symptoms. However, the precise pathology of how and why an unwanted immune reaction is triggered in certain individuals is still not well understood and also other stressful circumstances such as trauma or surgery seem to provoke GBS.

Citation: Fortuin AW, Kooi ALF, Mulder LJMM, Avoort IAM (2021) Guillain-Barré Syndrome Complicating First Trimester Pregnancy: A Case Report and Review of Literature. Obstet Gynecol Cases Rev 8:194. doi.org/10.23937/2377-9004/1410194

Accepted: March 16, 2021: Published: March 18, 2021

Copyright: (c) 2021 Fortuin AW, et al. This is an open-access article distributed under the terms of the Creative Commons Attribution License, which permits unrestricted use, distribution, and reproduction in any medium, provided the original author and source are credited. 
In Europe, the prevalence of GBS is 1-3/100.000 [5]. There are different subtypes, the Acute inflammatory demyelinating polyradiculoneuropathy (AIDP) being the most common [6]. It can occur at any age and is slightly more common in men. Diagnosis is made based on clinical examination and supported by Cerebral spinal fluid (CSF) analysis and electromyography. Imaging techniques may be helpful to rule out GBS mimics but are not useful to make the diagnosis.

Plasma exchange (PE) and Intravenous immunoglobulin (IVIG) are both proven effective treatments. Because of the minor side effects and non-invasive way of treatment, IVIG has become the first choice treatment in Europe, given in a dosage of $2 \mathrm{~g} / \mathrm{kg}$ in 2-5 days [5]. An ongoing trial is investigating the benefit of a second course of IVIG, given shortly after the first course, in severely affected patients [7]. The administration of high dose methylprednisolone as add-on therapy to IVIG does not result in significant improvements after four weeks, unless the outcomes are adjusted for some factors that are known to affect the prognosis of disease [8]. Therefore, in some health care centres, it is recommended to treat patients with methylprednisolone 500 $\mathrm{mg} /$ day for five days, starting within 48 hours from the start of treatment with IVIG, unless this is contra-indicated.

Guillain-Barré syndrome diagnosed during pregnancy is rare, with the prevalence being the same as in the general population. GBS can be easily missed in the first trimester due to its nonspecific complaints such as numbness and weakness of the limbs, which can erroneously be attributed to pregnancy or psychological complaints. Misinterpretation of the complaints can lead to delay in diagnosis and treatment. There are no specific guidelines for the treatment of GBS in pregnancy, most important being supportive care and close monitoring of autonomic functions and fetal growth. On its own, GBS is not an indication for termination of the pregnancy. We report a 26-year-old nullipara who developed GBS in the first trimester of pregnancy. In addition, we present an overview of the literature of the last twenty years, focusing on the given treatment, maternal as well as fetal outcomes and pathogens associated with GBS of women diagnosed in the first trimester of pregnancy.

\section{Methods}

\section{Search strategy}

To provide an overview of the first trimester pregnancies complicated by GBS, we identified relevant articles, published after January 2000 and up until January 2020 , by systematically searching Embase and Web of science. Details of the full search strategy are included in Appendix S1.

\section{Inclusion \& exclusion criteria}

We included articles describing women who were di- agnosed with GBS in the first trimester of pregnancy. Papers discussing Zika virus and anaesthesiological papers discussing anaesthetic management were excluded as these were beyond the scope of our research questions.

\section{Study selection}

Two independent investigators (AWF and ALFvdK) reviewed all titles and abstracts, and independently selected potentially eligible studies. Abstracts and reviews were excluded and only studies published in English were selected for the analysis. In case of disagreement, a third investigator was available to reach consensus. Full text papers were retrieved to assess fulfilment of the selection criteria (Figure 1). Cross-reference check was performed to identify additional studies that were potentially overlooked during the initial search.

\section{Results}

\section{Case report}

A 26-year-old gravida 2 para 0 presented with progressive sensibility problems of the lower limbs and loss of balance. As she had just found out she was pregnant, her general practitioner referred her to the OB/GYN outpatient clinic, where she was seen the next day. Her medical history showed a gastric bypass 5 years ago and irritable bowel syndrome. An earlier pregnancy, which had ended in an abortion, had been complicated by hyperemesis gravidarum. At presentation, an ultrasound indicated that she was in her $7^{\text {th }}$ week of pregnancy. She was immediately referred to the neurologist as her complaints were unlikely a direct result of her pregnancy.

The patient complaints included dysesthesia in her lower extremities and hands for one week that eventually extended to her mouth and tongue, which caused an unclear speech. Anunstable gait was present due to muscle weakness of the legs since one day. She experienced pain in her upper extremities and toes. Medical history did not reveal a previous illness. Travel history recorded recent trips to Aruba in the Caribbean Sea and Spain.

Neurological examination showed no abnormalities of the cranial nerves. Upper extremities showed distal muscle weakness, score 4 using the Medical research council (MRC) scale and a sensibility loss of both arms, proximal being more extended. Lower extremities showed proximal and distal weakness with MRC score 4 and sensibility loss of both legs. Hyporeflexia was present in both upper and lower extremities. It took her a lot of effort to get up from her chair and her gait was broadbased and unstable. Laboratory results showed slightly increased Creatinine kinase (CK) without further abnormalities. She was admitted to the neurology unit for monitoring and further evaluation. Six hours later, progressive muscle weakness, shortness of breath (without respiratory insufficiency) and difficulty swallowing was observed, which correlates with an Erasmus GBS respi- 


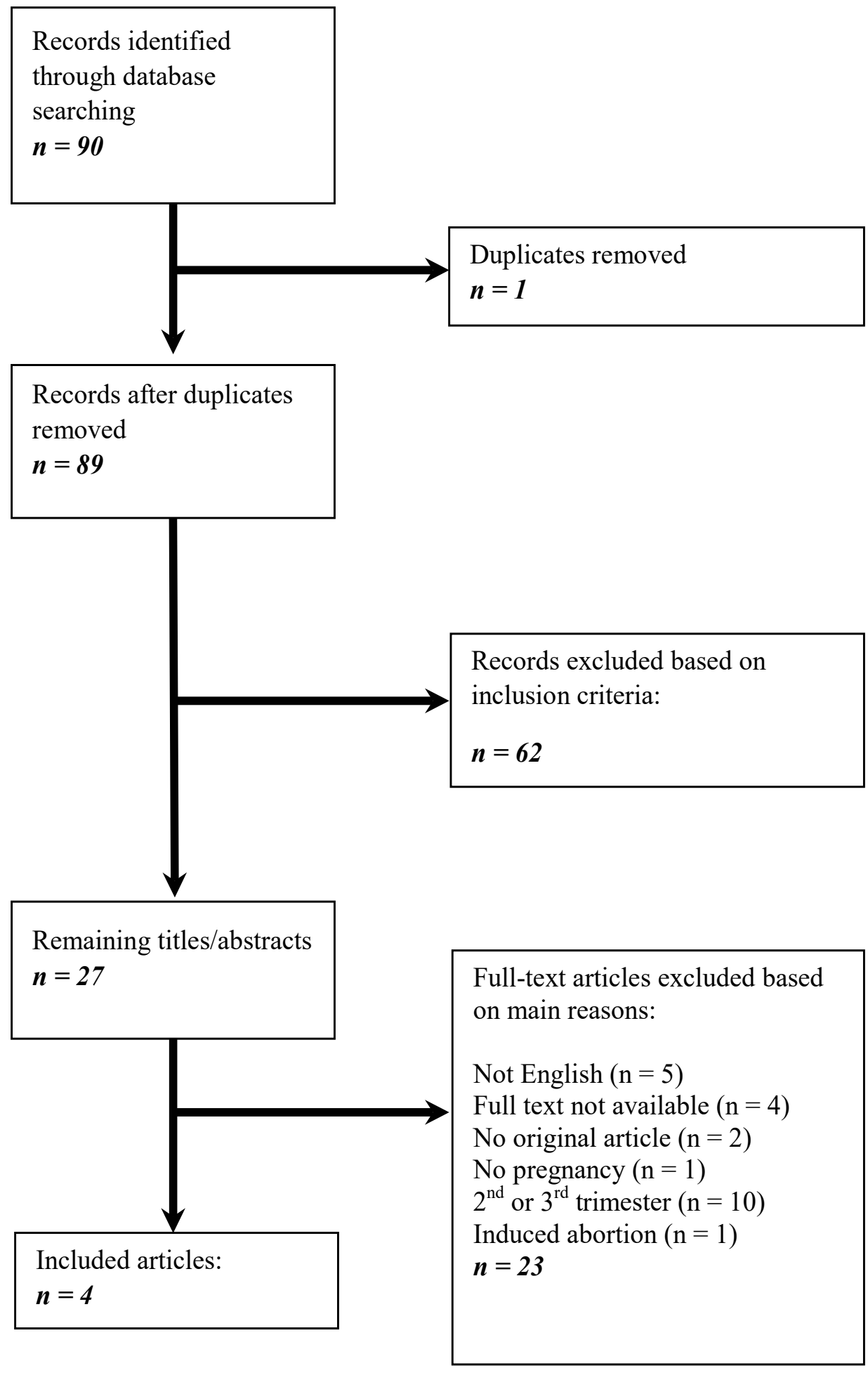

Figure 1: Flowchart of study selection process.

ratory insufficiency score (EGRIS) score of 3, giving a risk of $15 \%$ of respiratory insufficiency in the first week after onset of symptoms [9]. At this point, the decision was made to start with a five-day treatment of IVIG (with a daily dosage of $400 \mathrm{mg} / \mathrm{kg}$ ). The Intensive care unit (ICU) was notified, in case progressive respiratory complaints would require ICU admission. Continuous ECG monitoring was implied, vital capacity measured daily and a bladder scan performed daily because of the possibility of developing autonomic dysfunction. She showed multiple short episodes of sinus tachycardia without other arrhythmias, her vital capacity remained stable (1200 $\mathrm{ml}$ ) and she did not develop urinary retention. Therefore, no further interventions were needed. However, the patient did lose her ability to walk independently and had to adjust her diet because of difficulty swallowing. Also, her speech deteriorated to a whisper because of bulbar weakness and respiratory problems.

Magnetic resonance imaging (MRI) of cerebrum and cervical myelum showed no abnormalities. Evaluation of Cerebrospinal fluid (CSF) showed elevated protein 
levels with a normal white blood cell count. Electromyography two weeks after onset of complaints showed demyelination, confirming the diagnosis of acute inflammatory demyelinating polyneuropathy, the most common variant of GBS.

Her symptoms plateaued after five days, and subtle improvements were noticeable the days after. The physiotherapist and speech therapist were consulted at admission and closely involved during her hospital stay. At the $11^{\text {th }}$ day of admission, she was transferred to a rehabilitation centre for the rest of her recovery. At that time, she had regained some muscle strength and could walk several meters with the support of two persons and walking aid. She was then in her $9^{\text {th }}$ week of pregnancy. After another 8 weeks she was discharged from the rehabilitation centre and continued intensive revalidation as an outpatient. At gynaecologic follow up at 28 weeks of pregnancy she still had an unstable gait and loss of strength in her arms, and she still experienced some mild symptoms at time of delivery and at the end of follow up.

The infectious aetiology of the GBS was evaluated using extensive serological tests including TORCH (Toxoplasmosis, Other agents, Rubella, Cytomegalovirus, and Herpes Simplex). This showed the presence of anti-CMV IgG and IgM antibodies, with an avidity of 0.78 , indicating a cleared infection at least 3 months ago. Epstein Barr virus, herpes simplex, Rubella and Parvovirus B19 showed past infections, the tests for syphilis, hepatitis $B$ and human immunodeficiency virus and toxoplasmosis were negative. As she had not recently travelled to an epidemic area for Zika virus, Zika was not evaluated.

With regard to her bariatric surgery five year prior to her pregnancy, we monitored her vitamin levels during pregnancy. Initially, her vitamin levels were adequate. She received vitamin $K$ supplements in her third trimester for four weeks and afterwards her blood levels remained adequate. Her weight had been stable since three years, and her weight gain was normal during pregnancy. We considered her bariatric surgery in the past as an unlikely cause of the development of her symptoms because of the five year time span and the fact she was in a stable condition.

Due to an anaemia with low ferritin levels despite supplements, intravenous iron infusion was administered in the third trimester. She was diagnosed with gestational diabetes for which dietary restrictions proved to be sufficient, and for which extra growth ultrasounds were performed.

Labour was induced using a Foley balloon catheter at 38 weeks of gestation because of suspected fetal macrosomia. She underwent an emergency caesarean section due to fetal distress after artificial rupture of the membranes at $3 \mathrm{~cm}$ dilatation. A boy of $3935 \mathrm{gram}\left(97^{\text {th }}\right.$ percentile) with an Apgar score of 3/7/8, pH 7.23, Base Excess -3.7 was delivered. No further cord blood analysis was performed. The infant was initially treated with penicillin and gentamycin for a possible infection, but as CRP and blood cultures remained negative, this was stopped two days later and his recovery was uneventful. Maternal postoperative recovery was complicated by symptoms of an ileus, and treated with laxatives. Four months post-partum, no signs of neurological relapse had occurred.

\section{Study selection for review}

The search strategy yielded 89 unique records (Figure 1). After screening titles and abstracts, 27 articles were selected for detailed evaluation of full texts. After full-text assessment, four articles remained that reported GBS diagnosed in first trimester pregnancy. For the purpose of the current review, we focused on the preferred treatment in first trimester pregnant females diagnosed with GBS, pathogens associated with GBS in European countries, fetal outcomes and risk factors that are associated with bad fetal outcomes.

\section{Study characteristics}

The four included articles are all case reports, describing the course of a pregnant woman diagnosed with GBS in the first trimester. Brooks, et al. [10] and Kokubun, et al. [11] report two cases, but only one meets our inclusion criteria. All women are between the age of 23-32 years-old and otherwise healthy, one of them primigravida. For a complete overview of study characteristics, see Table 1.

\section{Outcomes}

All four described patients complained of bilateral,

Table 1: Study Characteristics of included articles.

\begin{tabular}{|l|l|l|l|l|}
\hline Article & Study design & Journal & Study size (n) & Patient characteristics \\
\hline Pollak, et al. [13] & Case report & BMJ & 1 & 23-year-old, G2P1 \\
\hline $\begin{array}{l}\text { JE Mendizabal, et } \\
\text { al. [12] }\end{array}$ & Case report & $\begin{array}{l}\text { Journal of Clinical } \\
\text { Virology }\end{array}$ & 1 & $\begin{array}{l}\text { 30-year-old, G3P1 } \\
\text { prednisone 0.3 mg/kg/d until the 12th } \\
\text { week of gestation, Because of a history of } \\
\text { spontaneous abortion and weakly positive } \\
\text { antinuclear antibodies }\end{array}$ \\
\hline Kokubun, et al. [11] & Case report & $\begin{array}{l}\text { Journal of Neurological } \\
\text { sciences }\end{array}$ & 2 (1 first trimester) & 32-year-old, Multiparous \\
\hline Brooks, et al. [10] & Case report & Anaesthesia & 2 (1 first trimester) & 28-year-old, G1P0 \\
\hline
\end{tabular}




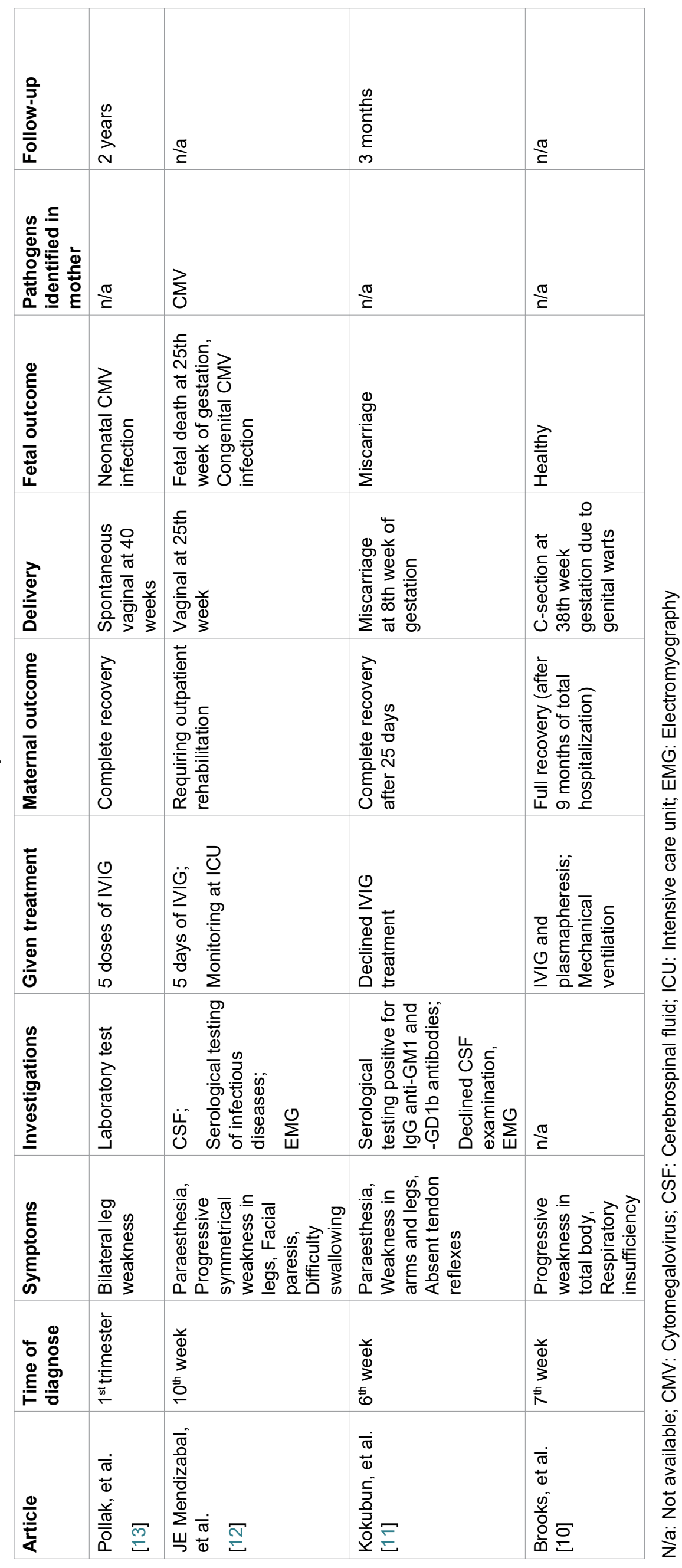


symmetrical weakness of the lower extremities, some of them accompanied by dysesthesia and extension to the upper extremities. In all cases, GBS was diagnosed based on clinical presentation. This diagnosis was supported by Cerebrospinal fluid (CSF) examination in one report and Electromyography (EMG) in two reports.

In the cases described by JE Mendizabal, et al. [12] and Brooks, et al. [10], admission to the Intensive care unit (ICU) was necessary for the monitoring of vital functions. One woman needed mechanical ventilation for 18 weeks because of respiratory muscle weakness.

The recommended treatment in all cases was IVIG. One patient rejected this therapy for unknown reasons and thus did not receive any treatment. In one patient, plasmapheresis was carried out in addition to IVIG treatment.

Monthly ultrasound monitoring was performed in one case, because of a maternal active CMV infection. The other articles did not describe an adjustment of the frequency of obstetrical controls.

As to the cause of the development of Guillain Barré syndrome, Pollak, et al. described Cytomegalovirus (CMV) as a possible causative agent, because of a neonatal infection of the new-born [13]. However, they did not carry out serological tests on the mother, and therefore other pathogens could also be responsible for the development of Guillain Barré syndrome. JE Mendizabal, et al. did find an active CMV infection in the mother, indicating this infection being the cause of her polyradiculoneuropathy [12].

All articles describe a complete recovery of the mother. One woman gave birth to a healthy baby. She had an elective Caesarean section for gynaecological reasons, unrelated to her GBS infection. Another infant was born with an active CMV infection and therefore was treated with platelet transfusion, antibiotics and long-term ganciclovir. At 2-year follow-up, the child had neurodevelopmental impairment with sensorineural deafness, poor language development and gross motor delay. The mother who denied treatment went on to have a miscarriage, however a relation with the GBS is not apparent. The last case reports an in utero fetal death (IUFD) at 25 weeks of ingestion, caused by chronic villitis based on a congenital CMV infection. For an overview of study results, see Table 2.

As in the other cases presented, the woman in our case report recovered adequately after treatment with IVIG and long lasting, intensive physical therapy.

\section{Discussion}

There are no specific guidelines for treatment of Guillain Barré syndrome in pregnancy and little is known about the pathogens that trigger this autoimmune polyradiculopathy, or fetal outcome after a maternal GBS infection.
We conducted a literature search to find articles describing GBS in first trimester pregnancies and found four publications of the past twenty years. Based on these articles, the management of GBS in pregnant women is similar to other patients and consists of intravenous immunoglobulins or plasmapheresis. Autonomic functions should be monitored and supportive care should be given when needed.

In this review, CMV was reported in two articles to be the infective agent that induced GBS. The other two articles did not describe testing for underlying maternal infections. This suggests that CMV is a significant cause of GBS in pregnant patients. A limitation of the study by Pollak, et al. [13] is the absence of testing for maternal active infections, leaving the possibility that GBS was induced by another agent. More articles need to be studied to give a reliable overview of the most common pathogens causing GBS in pregnant women. In the patient we describe, an infection with CMV had occurred, but at least 3 months prior to presentation, limiting the likelihood of this being the causative infection.

Primary CMV infection occurs in $0.5-4 \%$ of all pregnancies [14]. Congenital CMV transmission rates are around $30 \%$ in women who acquire primary CMV infection during pregnancy, while rates are well below $2 \%$ in women with a recurrent infection [15]. Approximately $10 \%$ of infants who acquire CMV in utero will have clinical signs at birth and are thus at high risk for severe sequelae $[15,16]$. Another $10-15 \%$ of infants who acquire CMV in utero are asymptomatic at birth but will develop long-term neurological sequelae, mostly primarily hearing loss [16]. Congenital CMV infection is more frequent with advancing gestation, but the severity of an infection is usually higher in cases of infection in early gestation $[17,18]$. The earliest signs of congenital CMV infection may be seen on routine fetal ultrasound at 20 weeks of gestation. Further evaluation, such as amniotic fluid testing, may be indicated in the presence of life-threatening symptoms in the fetus. Although there is currently no maternal antiviral treatment evidence-based effective for the fetus, antiviral treatment can be initiated postnatally for improved neurodevelopmental and audiological outcomes [19].

In the reported first trimester pregnancies complicated by Guillain-Barre syndrome, maternal CMV infection was indeed associated with adverse fetal outcome, causing neurological development problems in one case and IUFD in another. The woman with the most severe polyradiculopathy, with the need for mechanical ventilation for 18 weeks because of respiratory insufficiency, delivered a healthy baby after an elective caesarean delivery. This implies that the severity of the polyradiculopathy does not relate to fetal outcome, but the underlying maternal infection can result in adverse outcomes. In one of the excluded articles, one woman was reported to opt for termination of pregnancy during 
GBS, although the exact motivation for this choice was not discussed [20]. CMV serology was not mentioned in this report.

In conclusion, the treatment of GBS in pregnant patients is similar to the treatment of other patients and consists of IVIG and supportive care. This treatment in early pregnancy is effective and safe for the unborn child. There is no reason to withhold IVIG as treatment of GBS in early pregnancy. Methylprednisolone can be considered, but should be avoided in the first trimester. As CMV infection is a well-known trigger of GBS, and fetal transmission is associated with adverse neurodevelopmental and audiologic outcomes, it should be evaluated as causative agent in first trimester pregnancies complicated by GBS, together with the other TORCH agents.

\section{Acknowledgments}

\section{Conflict of interest}

The authors declare that they have no conflict of interest.

\section{Ethical approval}

This article does not contain any studies with animals nor humans performed by any of the authors.

\section{Informed consent on participation and publishing}

Obtained from the one participant in this case report.

\section{Authors' contributions}

Fortuin, Ikazia Hospital, Rotterdam, The Netherlands: Data analysis; Manuscript writing; Van der Kooi, Ikazia Hospital, Rotterdam, The Netherlands: Data analysis; Manuscript writing; Mulder, Ikazia Hospital, Rotterdam, The Netherlands: Manuscript writing; Van der Avoort, Ikazia Hospital, Rotterdam, The Netherlands: Manuscript writing

\section{Funding}

No funding was received for this study.

\section{References}

1. Berg VD, Walgaard C, Drenthen J, Fokke C, Jacobs BC, et al. (2014) "Guillain-Barre syndrome: Pathogenesis, diagnosis, treatment and prognosis. Nat Rev Neurol 10: 469-482.

2. Albert LJ, Inman RD (1999) Molecular mimicry and autoimmunity. N Engl J Med 341: 2068-2074.

3. Hadden RD, Karch H, Hartung HP, Zielasek J, Weissbrich B, et al. (2001) Preceding infections, immune factors, and outcome in Guillain-Barre syndrome. Neurology 56: 758-765.

4. Cao-Lormeau V-M, Blake A, Mons S, Lastere S, Roche C, et al. (2016) Guillain-Barre syndrome outbreak caused by Zika virus infection in French Polynesia. Lancet 387: 15311539.

5. Willison HJ, Jacobs BC, van Doorn PA (2016) Guillain-Barre syndrome. The Lancet 388: 717-727.
6. WALLING (2013) Guillain-barre syndrome. American Family Physician.

7. Walgaard C, Jacobs BC, Lingsma HF, Steyerberg EW, Cornblath DR, et al. (2018) Second IVIg course in Guillain-Barre syndrome patients with poor prognosis (SID-GBS trial): Protocol for a double-blind randomized, placebo-controlled clinical trial. J Peripher Nerv Syst 23: 210-215.

8. Koningsveld RV, Schmitz PIM, Van Der Meche FGA, Visser LH, Meulstee J, et al. (2004) Effect of methylprednisolone when added to standard treatment with intravenous immunoglobulin for Guillain-Barre syndrome: Randomised trial. Lancet 363: 192-196.

9. Walgaard C, Lingsma HF, Ruts L, Drenthen J, van Koningsveld $R$, et al. (2010) Prediction of respiratory insufficiency in Guillain-Barre syndrome. Ann Neurol 67: 781-787.

10. Brooks H, Christian AS, May AE (2000) Pregnancy, anaesthesia and Guillain Barre syndrome. Anaesthesia 55: 894898.

11. Kokubun N, Shahrizaila N, Hirata K, Yuki N (2013) Reversible conduction failure is distinct from neurophysiological patterns of recovery in mild demyelinating Guillain-Barre syndrome. J Neurol Sci 326: 111-114.

12. Mendizabal JE, Bassam BA (2016) Guillain-Barre syndrome and cytomegalovirus infection during pregnancy. $J$ Clin Virol 79: 74-76.

13. Pollak-Christian E, Lee KS (2016) Importance of diagnostic work-up of Guillain-Barre syndrome in pregnancy. BMJ Case Rep.

14. Nigro G (2009) Maternal-fetal cytomegalovirus infection: From diagnosis to therapy. J Matern Fetal Neonatal Med 22: $169-174$

15. Kenneson A, Cannon MJ (2007) Review and meta-analysis of the epidemiology of congenital cytomegalovirus (CMV) infection. Rev Med Virol 17: 253-276.

16. Dollard SC, Grosse SD, Ross DS (2007) New estimates of the prevalence of neurological and sensory sequelae and mortality associated with congenital cytomegalovirus infection. Rev Med Virol 17: 355-363.

17. Pass RF, Fowler KB, Boppana SB, Britt WJ, Stagno S (2006) Congenital cytomegalovirus infection following first trimester maternal infection: Symptoms at birth and outcome. J Clin Virol 35: 216-220.

18. Chatzakis C, Ville Y, Makrydimas G, Dinas K, Sotiriadis A, et al. (2020) Timing of primary maternal cytomegalovirus infection and rates of vertical transmission and fetal consequences. Am J Obstet Gynecol 223: 870-883.e11.

19. Davis NL, King CC, Kourtis AP (2017) Cytomegalovirus infection in pregnancy. Birth Defects Res 109: 336-346.

20. Zeeman GG (2001) A case of acute inflammatory demyelinating polyradiculoneuropathy in early pregnancy. Am J Perinatol 18: 213-215. 


\section{Appendix}

Appendix S1: Details of search strategy.

((((("guillain-barre syndrome"[MeSH Terms] OR ("guillain barre"[All Fields] AND "syndrome"[All Fields])) OR "guillain barre syndrome"[All Fields]) OR (("guillain"[All Fields] AND "barre"[All Fields]) AND "syndrome"[All Fields])) OR "guillain barre syndrome"[All Fields]) AND "pregnan*"[All Fields]) NOT (((((("zika virus"[MeSH Terms] OR ("zika"[All Fields] AND "virus"[All Fields])) OR "zika virus"[All Fields]) OR "zika"[All Fields]) OR "zika virus infection"[MeSH Terms]) OR (("zika"[All Fields] AND "virus"[All Fields]) AND "infection"[All Fields])) OR "zika virus infection"[All Fields])

\section{Translations}

guillain barre syndrome: "guillain-barre syndrome"[MeSH Terms] OR ("guillain-barre"[All Fields] AND "syndrome"[All Fields]) OR "guillain-barre syndrome"[All Fields] OR ("guillain"[All Fields] AND "barre"[All Fields] AND "syndrome"[All Fields]) OR "guillain barre syndrome"[All Fields]

Zika: "zika virus"[MeSH Terms] OR ("zika"[All Fields] AND "virus"[All Fields]) OR "zika virus"[All Fields] OR "zika"[All Fields] OR "zika virus infection"[MeSH Terms] OR ("zika"[All Fields] AND "virus"[All Fields] AND "infection"[All Fields]) OR "zika virus infection"[All Fields] 